\title{
Experienced Practitioners' Views on Interpersonal Skills in Telehealth Delivery
}

\author{
Beverly W. Henry \\ Northern Illinois University, bwhenry@niu.edu \\ Leah J. Ames \\ Northern Illinois University \\ Derryl E. Block \\ Northern Illinois University \\ John A. Vozenilek \\ OSF Healthcare System, Peoria, Illinois, USA
}

Follow this and additional works at: https://nsuworks.nova.edu/ijahsp

Part of the Medicine and Health Sciences Commons

\section{Recommended Citation}

Henry BW, Ames LJ, Block DE, Vozenilek JA. Experienced Practitioners' Views on Interpersonal Skills in Telehealth Delivery. The Internet Journal of Allied Health Sciences and Practice. 2018 Jan 01;16(2), Article 2.

This Manuscript is brought to you for free and open access by the College of Health Care Sciences at NSUWorks. It has been accepted for inclusion in Internet Journal of Allied Health Sciences and Practice by an authorized editor of NSUWorks. For more information, please contact nsuworks@nova.edu. 


\title{
Experienced Practitioners' Views on Interpersonal Skills in Telehealth Delivery
}

\begin{abstract}
Background. As telehealth becomes a larger part of the health care landscape, clinicians are becoming prepared to operate technology-based systems for conducting routine care and exchanging information. Less defined are interpersonal skills for telehealth care delivery such as communication and therapeutic relationships that can influence clinical outcomes. Examples include clinician adaptability to the communication process via telehealth, clinician congeniality in communications, and striving to achieve telepresence. The purpose of this study was to describe interpersonal skills for telehealth delivery to assist in the preparation of health professionals.
\end{abstract}

Methods. Aqualitative methods approached was used to build on results from our previously published systematic review. Semi-structured, in-depth interviews were conducted with experienced practitioners or educators $(n=6)$ at multiple regional telehealth centers. Video/audio-recorded sessions were transcribed verbatim and researchers conducted thematic analysis of data until achieving saturation of data.

Results. Participants provided their perspectives about interpersonal skills important for quality telehealth delivery based on professional experiences. Analysis of responses across interviews showed strong alignment with the six themes identified previously as non-technical clinician attributes: Preinteractional, Verbal Communication, Non-Verbal Communication, Relational, and Environmental. Also, an additional theme of Management/Operations emerged. Suggested training topics crossed clinical disciplines and ranged from telemedicine etiquette and verbal skills to equipment operation and billing and coding. Each study participant commented on benefits from preparation of telehealth clinicians related to the clinicianpatient interaction such as: patient engagement, patient-centered care, patient satisfaction, patient implementation of care plans, effective communication with patients, and quality assessment of telehealth sessions.

Conclusions. This study identified interpersonal skills that may be applied in professional education for telehealth delivery from the perspective of experienced practitioners. Further research could explore outcomes from professional preparation for interpersonal skills and patient perspectives.

\section{Author Bio(s)}

Beverly W. Henry, PhD, RDN, is Associate Dean for Academic Affairs and Professor of Nutrition and Dietetics in the College of Health and Human Sciences at Northern Illinois University, DeKalb, Illinois, USA. She is also a licensed dietitian in the state of Illinois.

Leah J. Ames, MS, MLS, is a doctoral student in the College of Health Sciences at Northern Illinois University, DeKalb, Illinois, USA. She is an experienced Medical Laboratory Scientist with a demonstrated history of working in a clinical laboratory and higher education.

Derryl E. Block, PhD, RN, is dean and professor in the College of Health Sciences at Northern Illinois University, DeKalb, Illinois, USA. Her research interests include health workforce development, public health nursing, and community development.

John A. Vozenilek, MD, FACP is the VP and Chief Medical Officer of Jump Simulation. He provides central coordination and oversight for OSF Healthcare's undergraduate, graduate, interdisciplinary, and continuing medical education programs.

\section{Acknowledgements}

The authors acknowledge with appreciation the participation by telehealth professionals interviewed for 
this study.

This manuscript is available in Internet Journal of Allied Health Sciences and Practice: https://nsuworks.nova.edu/ijahsp/vol16/iss2/2 


\title{
TUAHSP \\ The Internet Joutnal of Allied Health Sciences and Practice
}

Dedicated to allied health professional practice and education

Vol. 16 No. 2 ISSN 1540-580X

\section{Experienced Practitioners' Views on Interpersonal Skills in Telehealth Delivery}

\author{
Beverly W. Henry, PhD, RDN ${ }^{1}$ \\ Leah J. Ames, MS, MLS ${ }^{1}$ \\ Derryl E. Block, PhD, RN ${ }^{1}$ \\ John A. Vozenilek, MD, FACP2
}

1. Northern Illinois University

2. OSF Healthcare System

United States

\begin{abstract}
Background. As telehealth becomes a larger part of the health care landscape, clinicians are becoming prepared to operate technology-based systems for conducting routine care and exchanging information. Less defined are interpersonal skills for telehealth care delivery such as communication and therapeutic relationships that can influence clinical outcomes. Examples include clinician adaptability to the communication process via telehealth, clinician congeniality in communications, and clinician commitment to achieve telepresence. The purpose of this study was to describe interpersonal skills for telehealth delivery to assist in the preparation of health professionals. Methods. A qualitative methods approach was used to build on results from our previously published systematic review. Semi-structured, in-depth interviews were conducted with experienced practitioners or educators $(n=6)$ at multiple regional telehealth centers. Video/audio-recorded sessions were transcribed verbatim and researchers conducted thematic analysis of data until achieving saturation of data. Results. Participants provided their perspectives about interpersonal skills important for quality telehealth delivery based on professional experiences. Analysis of responses across interviews showed strong alignment with the six themes identified previously as non-technical clinician attributes: pre-interactional, verbal communication, non-verbal communication, relational, environmental, and educational. Also, an additional theme of management/operations emerged. Suggested training topics crossed clinical disciplines and ranged from telemedicine etiquette and verbal skills to equipment operation and billing and coding. Each study participant commented on benefits from preparation of telehealth clinicians related to the clinician-patient interaction such as patient engagement, patient-centered care, patient satisfaction, patient implementation of care plans, effective communication with patients, and quality assessment of telehealth sessions. Conclusions. This study identified interpersonal skills that may be applied in professional education for telehealth delivery from the perspective of experienced practitioners. Further research could explore outcomes from professional preparation for interpersonal skills and patient perspectives.
\end{abstract}

\section{INTRODUCTION}

It is estimated that 15 million Americans received medical care remotely in 2015, and health care executives expect further growth and new technologies.1,2 Key elements for clinical support using various types of information and communication technologies (ICTs) to improve health outcomes expanded beyond the physician role commonly referred to in the past as telemedicine.3,4 Clinicians from multiple disciplines provided care via telehealth at hospitals, clinics, and other sites such as nursing homes. The broader term, telehealth, is used in this research and encompasses multiple applications across disciplines for long-distance clinical activities, as well as health-related education, public health and health administration. ${ }^{4,5}$

With telehealth becoming more common, planning and support for service delivery needs to extend beyond technological advances to overcome obstacles. Currently, insurance coverage/reimbursement and regulatory systems remain problematic; policy changes may help address these issues to promote adoption of telehealth., 1,6,7 For example, the U.S. Department of Defense determined in 2016 that criteria for a medical visit no longer required that the patient and clinician be in the same physical location. ${ }^{8}$ Other key obstacles to optimizing use of telehealth include hesitation on the part of both clinicians and patients and perceptions about the 
disruptive nature of technology. ${ }^{2}$ To inform clinicians and managers, global and national groups provided general practice guidelines, as well as condition-specific recommendations to improve the utility of services for the remote delivery of health care. 7,9 The federally funded Consortium of Telehealth Resource Centers (TRCs) provides general guidelines for the education and practice of health care professionals including operational strategies to inform patients about the nature of telehealth services, suggestions for the physical environment, and recommendations for making referrals. This organization supports 12 regional centers to expand the availability and quality of health services via telehealth. ${ }^{10}$

Given the rising demand for telehealth services, one might expect a proportionate response in professional preparation programs across clinical disciplines. However, there is limited information about learning activities for health professionals using ICTs. Edirippulige and Armfield reviewed the literature in an effort to report education and training efforts that support integration of telehealth into routine care.11 Using the Preferred Reporting Items for Systematic Reviews and Meta-Analyses (PRISMA) guidelines, the authors identified only nine relevant studies. These nine studies were descriptive rather than experimental in nature. Results of their systematic review indicated telehealth education and training was provided by universities, as well as public and private organizations. The main topic areas included technological aspects, terminology, clinical applications, and the evidencebase for the effectiveness of telehealth. Information about how the telehealth medium of care delivery influenced clinician-patient communication was lacking.

In contrast, Miller proposed a conceptual model for telemedicine and doctor-patient communication that outlined characteristics of the patient, the provider, and the context of the medical encounter.12 In addition to the mode of service delivery, contextual characteristics such as culture, location, setting, organization, length of acquaintance, and the presence of a third party during the visit appeared to impact communication. Factors encompassed by Miller's model were verbal and non-verbal behaviors, such as information giving and seeking, social conversation, and relationship building between clinician and patient.

There is increasing emphasis on quality attributes for telehealth that include human as well as operational factors. LeRouge et al proposed four categories of attributes to achieve quality videoconferencing. ${ }^{13}$ Three categories were operationally labeled as technology, usability, and physical environment. ${ }^{13}$ The authors labeled the fourth category as human element attributes. Human factors included clinician adaptability to the process, clinician congeniality, and a recommendation to strive for telepresence to achieve quality care. LeRouge and colleagues confirmed their findings in a study of patient perspectives of telemedicine quality that highlighted the human aspect as well as technology and the physical environment. ${ }^{14}$ For those patients, highly-rated quality attributes of telehealth involved practical provider activities: clear directives, medical team coordination, professionalism, and telepresence. Esser and Goossens developed a framework for telehealth delivery that encompassed three perspectives: doctorpatient communication; technology acceptance; and technology-mediated communication. ${ }^{15}$ Technology-mediated communication drew from three communication theories: compensatory adaptation process, the social presence theory, and the media richness theory.

Through a systematic review of the telehealth-related literature on education and communication for health professionals in telehealth, this research team aimed to identify desirable practices for the development of communication skills, professionalism, and clinician-patient interactions. ${ }^{16}$ That study constituted the first phase of this research project and the results are briefly introduced here. Following PRISMA guidelines, search methods initially identified 5261 records that narrowed to 293 articles eligible for review regarding health care provider preparation or proficiency with interpersonal skills in telehealth care delivery. Then, qualitative analysis of the 45 articles accepted for final review revealed only general patterns or guidelines for preparation of health professionals, and the studies were largely observational in nature. The six themes that emerged were 1) clinician perceptions about telehealth; 2) interactions between clinician and patient including verbal communication, 3) non-verbal communication, and 4) relationship-building; 5) environmental attributes; and 6) guidelines for education interventions or evaluation of behaviors. While some educational activities and assessment tools were identified, evidence of the application of these efforts to improve proficiency with communication in telehealth delivery was lacking. Clearly, the limited results of that review confirmed a gap in understanding the best practices for pre-professional and continuing clinician education for effective communication in telehealth care delivery. This paper reports on the second phase of this research. The purpose of this study was to describe interpersonal skills important for quality telehealth delivery from the perspective of experienced telehealth practitioners and trainers. This information may highlight promising practices to support education of health professionals to improve preparation for, implementation of, and assessment of telehealth.

\section{METHODS}

\section{Study Design}

Given a lack of information about processes that develop clinicians' interpersonal skills for telehealth care delivery, a qualitative methods was used to build upon results from the systematic review. ${ }^{17}$ Semi-structured, in-depth interviews were conducted to

(C) The Internet Journal of Allied Health Sciences and Practice, 2018 
discover the perspectives of experienced individuals at multiple sites..$^{18}$ Thematic analysis of data followed guidelines described by Boyatzis. ${ }^{19}$ Sample size was considered sufficient when informal redundancy or saturation of the data was achieved. Then, results were compared with data from the previously reported systematic review. ${ }^{16}$ The plan to integrate multiple forms of data, both qualitative and quantitative, in sequential phases followed mixed methods research in a multiphase design as described by Creswell and Plano..$^{20}$ For this project, the quantitative systematic review in the first phase provided data for review through the qualitative methods in this second phase. Specifically, practitioner views were compared with information and skills in telehealth delivery that emerged from the systematic literature review. Approval was obtained from the institution's Office of Research Compliance and Integrity, and each participant provided informed consent prior to participation.

\section{Study Participants}

Participants were recruited from the Consortium of Telehealth Resource Centers. These centers are federally funded by the Office of the Advancement of Telehealth, Health Resources and Services Administration, and the Department of Health and Human Services. Study inquiries were sent using website contact information (general or individual) to the twelve regional centers. To be eligible for study inclusion, professionals could be of any discipline and needed to meet at least one of the following criteria: two years working in telehealth for at least eight hours per week, management of telehealth programs, or educator of clinicians about telehealth.

\section{Data Collection}

Data gathered through interviews followed a semi-structured format that included a pre-determined set of open-ended questions that allowed the interviewer to follow threads within a dialog between interviewer and participant. Questions focused on the participant's preparation to use telehealth, comparisons with face-to-face interactions, and unique, specific skillsets necessary to conduct successful telehealth sessions. The question guide is shown in Table 1 and includes seven items that form the basis of what participants were asked. ${ }^{21}$ One researcher (AA) recruited for this phase of the research conducted the interviews through virtual meetings using the Adobe Connect web conferencing software. The researcher sent each site a formal recruitment letter and subsequently made interview arrangements or received recommendations for participants that met the target sample description at that site. An email was sent to each participant prior to the individually scheduled interview time with instructions for accessing the Adobe Connect forum, a consent form, back-up contact information, and the Interview Guide (see Table 1) to allow participants to prepare. At the beginning of each interview meeting, participants received an introduction to the purpose of the study. With this background, the interviewer clarified three main goals: 1) to learn from the participant's professional experiences and opinions; 2) to gather information about non-technical behaviors, that is, clinician behaviors unrelated to clinical skills specific to an occupational discipline or operating technical system; and 3) to inform development of future educational activities for improving non-technical behaviors in telehealth. The audio portion of each interview was transcribed verbatim with notations of particular participant facial expressions and hand gestures.

Table 1. Interview Guide for Exploring TeleHealth Use

1. Please tell me your occupational title and how long you have been practicing in this field.

2. Reflecting back to your very first telehealth session(s) with you as the primary provider, can you describe how you prepared, either on a personal level or as supported by your organization?

3. Based on your own experiences what parts of telehealth do you find very different from traditional face-to-face care? I would like to focus on the interactions between you and the recipient.

4. Could you briefly describe some problems that you have encountered while delivering telehealth? I'm most interested with interaction problems between you and a recipient on the other end. Problems with the patient, language or communication barriers, trust, rapport, etc. rather than issues with clinical procedures or technology capabilities.

5. Have you ever taught or explained to someone how to deliver care via telehealth?

6. To wrap up our interview, is there anything else we should be thinking about when trying to identify competencies and important skills for telehealth delivery?

7. Do you have any general comments and suggestions about the research study? 


\section{Data Analysis}

Two researchers (AA, BB) independently conducted thematic analysis of interview data in a deductive process using the constant comparative method. ${ }^{18,19}$ They reviewed the interview transcripts, watched the recorded sessions, and identified data according to six pre-determined categories based on the themes from the systematic review. ${ }^{16}$ When conducting the initial coding of each transcript, the researchers (AA, BB) were blinded to the results of the other's analysis. To assess the appropriateness of labeling the coded data into these categories and assess the relevance of unlabeled data, the two analysts met periodically. The two analysts compared their independent appraisals of each transcript, cross-checked coding categories, revised the description of each theme as needed, and developed consensus. Upon reviewing results from the first interview, the research team met to discuss progress of the study including coding categories drawn from the themes and key findings of the systematic review. Through these discussions, the team determined that a seventh data category, Management/Operations, was needed to fully encompass the interview responses. (See Table 2 for the finalized coding scheme.) Then both analysts (AA, BB) independently reviewed the remaining transcripts and labeled data. The analysts met periodically to discuss each transcript with labeled data to develop consensus while later interviews were being conducted. After analyst review of the analyzed transcripts, the labeled data was extracted from the transcripts, and the excerpts were reorganized to identify theme-related categories. These worksheets were reviewed by two researchers (DD, BB) independently and jointly to confirm the appropriateness of the categories and to identify connections between the interviews. Lastly, a confirmatory review of data within categories and prevailing themes was done by a researcher $(\mathrm{AA})$.

Table 2. Coding Scheme for Thematic Analysis of Interview Transcripts

Theme 1. Pre-interactional

- Attitudes - seeing benefits to telehealth; openness, willingness, flexibility

- Competencies - define what competency means; professional conduct; manage technology

- Cultural awareness - recognize locale and population; specific culture/region, e.g. local news

Theme 2. Communication Verbal

- Behavioral skills - listening; phone communication; comprehensible/clear language; instrumental behaviors e.g. information seeking; empathetic language

- $\quad$ Timing - flow of communication; duration; pace

- Types of clinician talk - tangential to the visit e.g. social conversation or self-disclosure; patient-centered; avoid clinician dominance

Theme 3. Communication Non-verbal

- $\quad$ Eye contact - gaze angle

- Visual cues - pay attention and ask for more information about client facial expressions

- Empathetic gestures - exaggerated motions

Theme 4. Relational

- Rapport - foster collaboration; make a connection; pleasant yet direct manner; intimacy varies with mode

- Relationship building - therapeutic interaction or alliance; convey benevolence and empathy; collaborative decisionmaking; negotiation

Theme 5. Environmental

- Physical surroundings - familiar; limited distractions on both ends and interruptions

- Privacy - limit number of participants; distance may help sense of safety

Theme 6. Educational

- Types - preprofessional and continuing development; knowledge, skills, and attitudes; communication training

- Impacts - skills and self-efficacy; readiness for practice; clinical outcomes; patient safety

- Assessment - tele-ments; rules for practice; competency list; communication standards

Theme 7. Management/Operations

- Operation of a telehealth system

- Development of distance care - procedures; policies; technology proficiency

\section{RESULTS}

Recruitment for participants in the interview phase of the research resulted in responses to the general study inquiry sent to the twelve telehealth resource centers from six sites. Participant locations included the following regions: Northeast, Mid-Atlantic, South, California, and Northwest, United States. Although seven professionals agreed to participate in the interview, one ultimately declined because of availability. The remaining six participants met the inclusion criteria for telehealth-related work experience with 
at least eight hours per week for a period of 3.5 to 20 years (average 11.5 years). They represented male and female evenly and were based in academic as well as clinical settings. See Table 3 for background information of each participant. The researchers determined that saturation of the data was achieved during analysis and decided that no further recruitment would expand study findings. The findings of this phase of the sequential mixed methods study included analysis of qualitative data from practitioner interviews by integrating the data into the coding system established by the results of a previously published systematic review. ${ }^{16}$

Table 3. Background Information of Telehealth Participants

\begin{tabular}{|l|l|l|}
\hline \multicolumn{1}{|c|}{ Credentials } & \multicolumn{1}{|c|}{ Role of the Interviewee in Telehealth } & \multicolumn{1}{|c|}{ Telehealth Experience Highlights } \\
\hline Physician & Family Medicine & Developed a telemedicine system \\
\hline Educator & Curriculum, Evaluation, Development & Developed a tele-OSCE $^{*}$ \\
\hline Physician & Family Medicine & School-based telemedicine program \\
\hline Physician & Psychiatry and Family Medicine & Psychiatric telemedicine services \\
\hline Nurse & Executive Director & Remote Patient Monitoring program \\
\hline Nurse & Telemedicine Trauma Coordinator & Telehealth for high-risk pregnancy \\
\hline
\end{tabular}

*use of ICTs to deliver Objective Structured Clinical Examinations (OSCEs) for a graduate medical education program

The interviews resulted in an in-depth dialogue between interviewer and participant with an average duration of 49 minutes (range of 26 to 75 minutes). Four interviews were successfully conducted using the Adobe Connect interface. Two interviews were conducted via telephone because of a scheduling conflict and computer problems. Given the participants' experience with technology, technical difficulties were manageable. Two participants experienced small delays waiting to download a Flash plugin on their computer for the Adobe Connect software.

The analysis of data collected through the interviews progressed as planned. Analysis of the interview transcripts confirmed the six theme-related categories decided a priori and the one added category of Management/Operations. The research team agreed upon the assignment of coded data into the seven categories and the list of categorized data from transcript excerpts included comments drawn from each participant within each category.

\section{Perspectives about Interpersonal Skills in Telehealth}

A final review of the transcripts and the coded data confirmed the three goals of the interviews were addressed. For the first goal, each participant drew from professional experience and opinion in his/her responses. This was determined by the interviewer and when reviewing transcripts. Participants frequently referred to their prior and current work experiences.

The second goal was attained as the focus of interviews remained on non-technical behaviors and interpersonal skills important for quality telehealth delivery. Quotes exemplifying these two goals are noted here.

"In the beginning, we only had training on how to use the technology of the vendor... and the equipment we used then had much more delay through a telephone line ... still need to be cognitive about making sure the person's mouth has stopped prior to talking...learning to get used to seeing yourself on a daily basis and trying to make that human connection."

"One of the first things we look at is etiquette. Things to remember because you are not in the room with the patient... you can't see what's going on outside of where the camera is pointing ... remember patient confidentiality." "There's a little bit different interaction with the patient ... without physical engagement ... that makes it more challenging to get to the same level of a relationship... I think people talk more in telehealth visits... patient engagement varies in telehealth... you work to bring out more responses - ask more questions."

"Once you get the equipment and you get a connection using telemedicine, if you are able to behave as if you are in the same room, patients will respond in kind...if you look like you're uncomfortable with the equipment and you are not prepared, it doesn't work as well... but if you just talk to the camera, making eye contact with the camera, I find it's not very hard to do."

For the third goal of the interviews, findings related to recommendations for educational activities to improve non-technical behaviors in telehealth, participants' comments showed more variability. On one hand, a participant said, "anybody who is going to touch this [telehealth] needs to be trained from the top down." Participant-suggested training topics ranged from telemedicine etiquette and verbal skills to equipment operation and billing and coding. Another said that one needed to "train nurses on how to interact and engage with patients ... on how to communicate with patients." Also, a participant proposed training largely for

(C) The Internet Journal of Allied Health Sciences and Practice, 2018 
technology, "if there's someone who's less comfortable with technology, then they'd require some tutoring." Several responses indicated their training was on-the-job: "We basically learned by trial and error." However, each participant commented on some benefit from training for telehealth clinicians that related to the clinician-patient interaction. These benefits included patient engagement, patient-centered care, patient satisfaction, patient implementation of care plans, effective communication with patients, and quality assessment of telehealth sessions.

Also, some participants shared information about resources such as the American Telemedicine Association and the need for future research. This quote was taken from the concluding comments of one participant.

"We need to explore areas of research besides diagnostic accuracy ... consider human factors ... how humans interact with machines or how humans interact with each other using machines. What's important in human factors in tele-mental health? What is important in human factors when you are doing home hospice via telemedicine?"

\section{Thematic Analysis of Data}

Thematic analysis conducted on the data within the six interviews revealed each participant provided statements related to all six pre-determined themes during the first phase of this research (reported elsewhere). ${ }^{16}$ In addition, study results were analyzed through a review of comments across interviews by the researchers. The research team members identified strong alignment of interview data with the six themes previously identified from the systematic review. Summary statements and participant quotes reflected responses within the six themes. Listed in Table 4 are representative quotes from this phase of the research confirming the six themes and related to the key findings.

Table 4. Participants' Comments within the Six Themes of Skill Areas for Telehealth Clinicians

Theme 1. Pre-interactional - things that make a difference to clinicians working in telehealth

- Awareness of patient benefit

"so it's provider buy-in [that matters]"

"the patients really appreciate not having to travel distances to see their provider ... and in many cases prefer it over a face-to-face encounter"

- Competence with technology

"includes a lot of cool technology, which I do like"

"if you express frustration [with technology], then you may absolutely turn off your patient, and that kills the opportunity to have a good interaction"

- $\quad$ Attitudes about this form of care delivery "need to be competent in this future practice model"

"telemedicine is not a different specialty, it's just a way of seeing patients"

Theme 2. Communication Verbal - these skills are very important and are generalizable

- Communication style

"the intangible quality of does that patient answer their questions ... a style thing ... the ability to direct the exam ... how do they end things? How do they make the patient feel it isn't 'okay, click - we're done"

"... patient says, 'I don't like her, I couldn't understand her"'

- Accommodations for equipment

"verbalize what he/she is doing when not focused on the patient"

"you have to figure out how not to step on the last sentence of the last person ... some hesitation ... there's lag time... all that requires patience"

- Telepresence

"some people have a better way of talking on TV, it's a bit of a mystery"

"deliver patient-centered care through the computer"

Theme 3. Communication Non-verbal - these behaviors link to verbal and relational and remain as a focus area

- Eye contact

"need to know how to make eye contact when looking at the camera"

"Because face-to-face, when you meet you can connect with your eyes."

- Body language

"a patient said, 'I don't like her, she was moving around. I don't trust her."'

"You can get immediate feedback from people, you can see their body language." 
- Screen etiquette

"having a camera presence is important as well"

"natural tele-presence to remember or know what they have to do ... a style ... you wouldn't even know that we are in two separate places"

Theme 4. Relational - connections possible with camera or phone between clinician and patient

- Rapport

"you are invading the patient's territory, it is important to be respectful"

"make them feel comfortable and it's like the camera disappears"

- Empathy

"I couldn't hold his hand when he started crying, I tried an empathetic connection."

"trying to make that human connection"

- Relationship

"spend more time building the relationship"

"not making the technology the point, making the patient feel cared for"

Theme 5. Environmental - the setting has pros and cons when you are not in the same physical location

- $\quad$ Setting

"the environment needs not to be disruptive, to support patient engagement"

"answer the patient's questions around the technology present"

- Confidentiality

"answer the patient's questions around confidentiality"

"ask if there is someone else in the room; is that okay for the discussion?"

- Virtual space

"sometimes the virtual relationship is better, patients may disclose more"

"I can zoom in without getting in their personal space"

Theme 6 . Educational - lots of suggestions about what could be done

- Types

"we started from scratch for school-based program, we share our materials"

"[need to] work with health plans and universities on how to train the next generation"

- Impact

"competency-based training might improve quality care measures"

"train to interact and engage so that patients follow the care plan"

- Assessment

"checklist items ... to assess their ability to deliver patient-centered care"

"there's the subjective approach, did the doctor treat you well?

For the seventh theme (Management/Operations), findings that emerged from the interview results included perspectives about telehealth management. Each participant referred to procedures or logistics, policies, or technology needed to support clinicianpatient interactions. Quotes exemplifying this finding are noted here:

"How does the presenter [the person who brings the patient to the visit] at the distant site enable and support it to make a visit successful?"

"We want to understand the workflow and determine how to integrate the steps of remote patient monitoring into that process."

"We need to address barriers such as reimbursement; are always working on policy changes."

"How do you develop sustainable programs?"

"We have a wonderful technical support system here so it didn't take much to get up and running."

\section{DISCUSSION}

Areas of interpersonal skills for care delivery via telehealth were explored in this study. Experienced practitioners were interviewed from six regional telehealth centers and participants spoke easily from their backgrounds in various disciplines and modes of telehealth delivery regardless of video or telephone interview connections. While the focus of the interviews remained on non-

(c) The Internet Journal of Allied Health Sciences and Practice, 2018 
technical clinician behaviors indicating human elements, such as clinician-patient interaction, respondents also included management and operational practices that may influence communication and rapport-building. The addition of this content may reflect the broadness of the term telehealth to include health administration, as well as clinical roles for these experienced practitioners.

The findings of this study reinforced the skills encompassed in Miller's model of doctor-patient communication to varying degrees. ${ }^{12}$ For example, the need to gather information about a patient's cultural background and locale when using telehealth was remarked upon in only three interviews, although the data category for cultural awareness was predefined from the literature review. In contrast, each study participant emphasized the importance of both verbal and non-verbal communication skills. Also, building a partnership between clinician and patients was referred to in the study participants' responses about rapport-building, communicating empathy, and developing relationships. Factors encompassed by Miller's model were verbal and non-verbal behaviors, such as information giving and seeking, social conversation, and partnership building. Study participants also commented that distance between clinician and patient may at times enhance patient disclosure. This is consistent with prior research that suggested patients can feel safer with the distance of telehealth.22,23 However, the absence of physical touch, described by some participants as challenging, may reinforce the need to prepare specifically for telehealth care as part of routine professional education.

The findings of this study relate to the literature on computer-mediated communication (CMC). For example, the media-naturalness theory portrayed how speech, facial expression, and body language may require different cognitive loads of telehealth participants. ${ }^{24}$ Our participants acknowledged that telecommunication can be harder than face-to-face communication. As described by Kock, the complex process of electronic communication with different modes of stimuli can require participants to estimate the meaning of messages through means other than those used in face-to-face communication. ${ }^{25}$ However, clinicians may get better at CMC through a compensatory adaptation process. Through compensatory adaptation, participants learn to modify their behaviors and their interpretation of verbal and non-verbal messages used in electronic communication. ${ }^{25}$ This suggests potential benefits to ongoing professional development for clinicians to use in their communication and to orient patients to telehealth. In addition, the media-richness theory may be applied. This theory considered media forms in terms of non-verbal, as well as verbal communication. ${ }^{26}$ This perspective supported the researchers' decision to keep skill building with verbal and nonverbal communication as two separate content areas during data analysis for this study.

The study findings also reinforced the importance of clinicians working to make connections with the patient as is described in the social presence theory. ${ }^{27-29}$ The social presence theory addressed how different mediums convey the presence of individuals to each other and how one might still be perceived as a real person via CMC. Within the social presence theory, components of interest included verbal information exchange and interpersonal contacts through non-verbal gestures, gaze, and body language. Participants of this study emphasized non-verbal behaviors including gaze, body language, and gestures. Although not mentioning computer-mediated communication specifically, participants of this study referred to the importance of communication skills that may be related to behaviors that help one to be perceived as a real person. They also raised the point of looking into the camera and communicating through virtual eye contact as if one could make the camera disappear through their communication with the patient. Collectively, their responses suggest that virtual connections can mimic face-to-face exchanges, though they can be more difficult and take effort as described by the media-naturalness theory and compensatory adaptation. ${ }^{24,25}$ The recommendation to strive for telepresence as a quality attribute by LeRouge et al was also reflected in the findings of this study. ${ }^{13,14}$ Participants referred to telepresence or screen etiquette as part of both verbal and non-verbal communication skills.

Interestingly, the participants attributed much of a clinician's attitude about telehealth delivery to two key issues: clinician buy-in and technology competence. Participants stated often that clinicians needed to accept that telehealth is going to be a routine tool for care delivery and that all need to be competent in this practice model. Study participants expanded on reasons for these views such as expectations for patient benefit and improved outcomes. To paraphrase the participants, telehealth is not a different specialty -- rather it is another way to see patients. In addition, participants commented to varying degrees about how a preference for or against technology affected clinicians' attitudes. For some it seemed that technology was what interested them in the first place, or influenced them growing into roles in telehealth programs. Others recognized that one must avoid showing frustration with technology to maintain good interactions and clinician effectiveness. In this phase of the research, cultural awareness of care delivery to individuals in a different physical location was commented on by some but not all of the participants.

These results are limited in the sample size and suggestions for professional development. The determination to stop data collection at the sample size of six was based on the depth of information provided by participants, frequency and intensity of responses about interpersonal skills in telehealth delivery, and the shared views across interviews. The study findings concurred with general guidelines for interpersonal skill development and specific applications to telehealth were provided by participants. However, the potential for bias exists from the researchers' perspectives being swayed by prior research. Bias was limited by

(C) The Internet Journal of Allied Health Sciences and Practice, 2018 
selecting an interviewer new to the project. Also, despite prompting to do so, these experienced participants rarely commented on formal assessment of learning activities to develop non-technical skills for telehealth. Clinicians newer to telehealth services might have different views about professional development needs. Also, the patient perspective about clinician interpersonal skills was considered only on a limited basis by study participants.

\section{CONCLUSION}

This study identified interpersonal skills that may be applied to health professional's telehealth practice from the perspective of experienced practitioners. These findings confirmed the previously reported themes of pre-interactional, verbal communication, non-verbal communication, relational, environmental, educational, and an added Management/Operations theme. ${ }^{16}$ The findings suggest that there is a gap in professional preparation for telehealth. Implications for practice may be drawn from the six themes for improving outcomes through professional preparation. In addition, further understanding of the policies, training, and operations for telehealth programs may be considered with professional preparation. Implications for further research include offering telehealth delivery as an extension of clinical skills development to support clinician skills in routine care delivery across disciplines. Finally, future studies could explore a key aspect of the communication partnership that was limited in the first two phases of this research: the patient perspectives of effective clinician interpersonal skills and telehealth delivery.

\section{REFERENCES}

1. Beck M. How telemedicine is transforming health care. Wall Street Journal. Available at https://www.wsj.com/articles/howtelemedicine-is-transforming-health-care-1466993402. Published June 26, 2016. Accessed June 8, 2017.

2. Closing the Telehealth Gap. Avizia website. Available at https://www.avizia.com/research-report-closing-telehealth-gap/. Published 2016. Accessed April 1, 2017.

3. World Health Organization [WHO]. Telemedicine: Opportunities and Developments in Member States: report on the second global survey on ehealth. 2010. Available at http://www.who.int/goe/publications/goe_telemedicine_2010.pdf. Acessed November 29, 2017.

4. Center for Connected Health Policy [CCHP]. What is Telehealth? Available at http://www.cchpca.org/what-is-telehealth. Accessed November 28, 2017.

5. Health Resources \& Services Administration [HRSA]. Telehealth Programs. Available at https://www.hrsa.gov/ruralhealth/telehealth/index.html. Accessed November 29, 2017.

6. Weinstein RS, Lopez AM, Joseph BA, Erps KA, Holcomb M, et al. Telemedicine, telehealth, and mobile health applications that work: opportunities and barriers. Am J Med. 2014;127(3):183-7. doi: 10.1016/j.amjmed.2013.09.032

7. The Innovation Working Group. Roadmap for telemedicine: key considerations and recommendations. 2014. Available at https://www.ghdonline.org/uploads/road_map_for_telemedicine_iwg_asia_2014.pdf. Accessed December 2, 2017.

8. Woodson, J. The Assistant Secretary of Defense memo. Provision of Telemedicine at a Patient's Location. Health Affairs Memo, 2016. Available at http://static.politico.com/9e/6a/2beca60849fb898946b436968e52/assistant-defense-secretarymemo-on-step-act.pdf. Accessed June 8, 2017.

9. The American Telemedicine Association (ATA). Practice Guidelines for Live, on Demand Primary and Urgent Care, 2014. Available at http://www.americantelemed.org/home. Accessed June 8, 2017.

10. Consortium of Telehalth Resource Centers. 2011-2017. Telehealth Resource Centers. Available at https://www.telehealthresourcecenter.org/overview/welcome-consortium-telehealth-resource-centers-website. Accessed November 29, 2017.

11. Edirippulige $S$, Armfield NR. Education and training to support the use clinical telehealth: A review of the literature. $J$ Telemed Telecare. 2016;23(2):273-82. [PMID: 26892005]

12. Miller EA. Telemedicine and doctor-patient communication: a theoretical framework for evaluation. $J$ Telemed Telecare. 2002;8:311-8. [PMID: 125379717]

13. LeRouge C, Garfield MJ, Hevner AR. Quality attributes in telemedicine video conferencing. Proceedings of the $35^{\text {th }}$ Hawaii International Conference on System Sciences. 2002. Available at https://www.computer.org/csdl/proceedings/hicss/2002/1435/06/14350159.pdf. Accessed June 8, 2017.

14. LeRouge CM, Garfield MJ, Hevner AR. Patient perspectives of telemedicine quality. Patient Prefer Adherence. 2014;9:2540. [PMID: 25565781]

15. Esser PE, Goossens RHM. A framework for the design of user-centered teleconsulting systems. J Telemed Telecare. 2009;15:32-9. [PMID: 19139218]

16. Henry BW, Block DE, Ciesla JR, McGowan BA, Vozenilek JA. Clinician behaviors in telehealth care delivery: a systematic review. Adv Health Sci Ed. 2016;22(4):869-88. [PMID: 27696102]

(c) The Internet Journal of Allied Health Sciences and Practice, 2018 
17. Creswell JW, Klassen AC, Plano Clark VL, Smith KC for the Office of Behavioral and Social Sciences Research. Best Practices for Mixed Methods Research in the Health Sciences. National Institutes of Health. Available at https://obssr.od.nih.gov/training/mixed-methods-research/. Published August 2011. Accessed June 8, 2017.

18. Bogdan R, Biklen SK. Qualitative Research for Education: An Introduction to Theory and Methods, 3e. Boston: Allyn and Bacon; 1998.

19. Boyatzis RE. Transforming Qualitative Information: Thematic Analysis and Code Development. Thousand Oaks, CA: Sage Publications; 1998.

20. Creswell JW, Plano Clark VL. Designing and Conducting Mixed Methods Research, 2e. Thousand Oaks, CA: Sage; 2011.

21. Edwards R, Holland J. What is Qualitative Interviewing? New York: Bloomsbury Academic; 2013;2-3.

22. Pols J. The heart of the matter. About good nursing and telecare. Health Care Anal. 2010;18:374-88. [PMID: 20063198]

23. Simpson SG, Reid CL. Therapeutic alliance in videoconferencing psychotherapy: a review. Au J Rural Health. 2014;22:28099. [PMID: 25495622]

24. Kock N. The psychobiological model: towards a new theory of computer-mediated communication based on Darwinian evolution. Organ Sci. 2004;15(3):327-48. doi: 10.1287/orsc.1040.0071

25. Kock N. Media naturalness and compensatory encoding: the burden of electronic media obstacles is on senders. Dec Sup Sys. 2007;44:175-87. doi:10.1016/j.dss.2007.03.011

26. Daft RL, Lengel RH. Organizational information requirements, media richness and structural design. Management Sci. 1986;32(5):554-71.

27. Short J, Williams E, Christie B. The Social Psychology of Telecommunications. New York: John Wiley and Sons Ltd; 1976.

28. Cukor $P$, Baer $L$, Willis $S$, Leahy $L$, O'Laughlin $J$, et al. Use of videophone and low-cost standard telephone lines to provide a social presence in telepsychiatry. Telemed. 1998;4(4):313-21. [PMID: 10220471]

29. McLaren P, Ball CJ. Telemedicine: lessons remain unheeded. Brit Med J. 1995;310(6991):1390-2. [PMID: 7787547] 